\title{
Safe operation as a social construct
}

\author{
GENE I. ROCHLIN* \\ Energy and Resources Group, 310 Barrows Hall, University of California at \\ Berkeley, Berkeley, CA 94720-3050, USA
}

Keywords: Safety; Social construction; Safety culture; Nuclear operations; Reliability; Agency.

\begin{abstract}
Empirical work on organizations that manage complex, potentially hazardous technical operations with a surprisingly low rate of serious incidents shows that operational safety is more than the management or avoidance of risk or error. Safety so defined is an ongoing intersubjective construct not readily measured in terms of safety cultures, structures, functions, or other commonly used descriptors of technical or organizational attributes that fail fully to take into account collective as well as individual agency. In the cases that the author has studied, it is represented by the interactive dynamic between operators and managers, as well as their engagement with operational and organizational conditions. The maintenance of safe operation so defined is an interactive, dynamic and communicative act, hence it is particularly vulnerable to disruption or distortion by well-meant but imperfectly informed interventions aimed at eliminating or reducing 'human error' that do not take into account the importance of the processes by which the construction of safe operation is created and maintained.
\end{abstract}

\section{Introduction}

When the Berkeley 'high reliability organization' (HRO) group first set out to examine the role of operators in maintaining safety in a variety of complex, hightechnology operations, they took as their baseline the 'normal accident' perspective that has become the norm for studies of complex and tightly coupled technical systems (Perrow 1984, La Porte 1996). The group therefore focused initially on indicators for judging how errors were avoided and risk thereby managed or controlled (Rochlin et al. 1987, Rochlin 1993, La Porte 1996). What was found instead was an operational state that represented more than avoidance of risk or management of error. Those organizations characterized as HROs all show a positive engagement with the construction of operational safety that extends beyond controlling or mitigating untoward or unexpected events and seeks instead to anticipate and plan for them. 'Safety culture' as described in the management literature is usually a systemic, structural and static construct (INSAG 1991, Pidgeon 1991). In contrast, the culture of safety that was observed is a dynamic, intersubjectively constructed belief in the possibility of continued operational safety, instantiated by experience with anticipation of events that could have led to serious errors, and complemented by the continuing expectation of future surprise. Rather

*e-mail: armsis@ socrates.berkeley.edu 
than taking success as a basis for confidence, these operators, and their managers, maintain a self-conscious dialectic between collective learning from success and the deep belief that no learning can be taken to be exhaustive because the knowledge base for the complex and dangerous operations in question is inherently and permanently imperfect.

The observations made are easier to describe and report than to analyse, since the existing literature provides little methodological, ontological or theoretical guidance. Although there are a broad range of studies of possibly similar circumstances reported in the literatures of social psychology, organization studies, human error analysis, cognitive science and cognitive ergonomics, most of it remains more descriptive of the processes of risk and error than of safety defined in terms other than risk management and error avoidance. Moreover, many or perhaps most of the more recent and powerful theories of organizational and institutional performance have either gained their breadth and theoretical integration at the expense of theories of agency (Christensen et al. 1997), or adopted a very restricted notion that fails to distinguish agency from action (Sewell 1992). The purpose of this paper is to set out the broader definition of safety that emerges from this work, and to argue that it cannot be readily understood unless and until a better theory for analysing the role of agency in shaping both the structure and the performance of complex, high-risk socio-technical operations is developed.

\section{Safety is more than the management of risk}

The literature on risk provides two sets of definitions, one primarily technical that focuses on the causes and consequences of operational error, and one largely social and political whose emphasis in on representation, perception and interpretation (Krimsky and Golding 1992). As is the case for risk, safety may also be defined formally or technically in terms of minimizing errors and measurable consequences, but it is more appropriate to adopt the principle used by Slovic (1992: 119) in his social studies of risk and note that safety does not exist 'out there' independent of our minds and culture, ready to be measured, but is a constructed human concept, more easily judged than defined. Moreover, it is not sufficient to construct interpretations of safety reflexively and negatively, e.g. as the absence of accident, the avoidance of error or even the control of risk. It was not difficult to obtain in the interviews reasonable agreement on how an outside observer could measure safety in terms of error and consequences; empirical indicators of the broader social and organizational definitions of safety were more difficult to obtain. When operators of reliable systems such as air traffic control centres or well-performing nuclear power plants specified a set of operating conditions as being 'safe', they were expressing their evaluation of a positive state of the operational subsystem mediated by specific forms of human action, and that evaluation reflexively became part of the state of safety they were describing.

The social science literature provides many different ways to express this form of interaction. It might be called a frame, a representation or a social construction, depending upon the model for analysing the relationship between the actors, their equipment, and the task at hand. Each of the relevant literatures was suggestive, but none were entirely satisfying as a mode of explanation for the HRO group's observations, and none supplied a theory of agency sufficiently rich to provide an explanatory framework that fully incorporated the dynamic role of the operators in the process. A great deal of the work on risk or error done in 
these modes expressed to some extent the idea that safety is an active construct, and not just a passive organizational state or structure, or a behavioural adaptation to technical circumstances, but none followed through. Burns and Dietz (1992: 208), for example, begin their review article on socio-technical systems by asserting: 'We emphasize agency'. However, when they analyse safety in nuclear power plant operations, they return to the normal accident model, which is explicitly structural and system-level, and in the process shift from thinking about safety to analysing sources of error and risk. (Burns and Dietz 1992: 217ff.). Cognitive ergonomics goes further in supplying a means to think about the operators and the machines they operate as integrated actors (Hutchins 1995, Hollnagel 1997), but having a theory of action is not the same as having a theory of agency (Emirbayer and Goodwin 1994).

The breadth of specification and performance of the several socio-technical systems under study argued against interpreting safety in technically- or industriallyspecific terms. For some of the organizations, there were types or classes of error or accident that could not be tolerated at all. Others performed tasks for which the complete absence or suppression of errors and accidents was not possible; they were judged to be safe because the measurable and observable rates of errors or nearmisses was so much lower than one would expect by comparing their performance with that typical of more conventional organizations (Weick and Roberts 1993). Although all of the organizations also had to maintain safety in the face of continuing demands on performance and schedule, those demands varied from organization to organization, and, to some extent from task to task. Akrich (1992) argues that actor analysis requires realizing that for every actor there is a position corresponding to a specific socio-technical network, and that no actor can be confined to a single position. The present work requires an extension of that approach to task groups and other organizational subunits such as operations teams. The position of any operational group as an actor is therefore situational and dynamically constructed, and evaluations of safety are not easily connected to 'objective' measures of real world performance.

Safety was even more difficult to specify in the most important domain of all, performance and behaviour in relation to self-constructed expectations and demands. Most operators reported that the chance of error or accident actually increased when those at the controls felt that they were in an 'unsafe state'. However even they were unable to provide a characterization of those conditions that would result in their making that judgement. They did not volunteer that the converse was true, i.e. that operations were more likely to be safe when operators were comfortable that they were operating safely, even though that is the logical consequence of the more negative statement. Indeed, they tended to avoid the subject completely, and to respond vaguely when asked about it. That is consistent with the finding that part of the construction of safety in these organizations is a tendency to put a negative connotation on any aspect that emphasizes responding through individual action instead of adapting through collective agency.

\section{A few salient properties}

It was clear that safety was in some sense not just an intersubjective construct, a story the operators were telling to each other about each other in search of common interpretations. It more closely resembled what Wiley (1988) has called 'generic subjectivity', the construction of a social culture that provides meaning and purpose 
to the systems processes that they operate or manage. How then do they differ from other organizations for whom professions of being safe might be better characterized as over-confidence and poor judgement or excessive caution (Simpson 1996)? In the author's view, the lack of self-promotion or aggressive self-confidence is in itself a useful gauge, as is the desire to attribute errors or mistakes to the entire organization (including its structure) as well as to human action. Nevertheless, the lack of a theory of agency means that further efforts to determine the nature of the relationship between perceptions of and beliefs in safe operations and measurable indicators of performance still remains outside the scope of comparative field work or categorical analysis, let alone projective or prescriptive theorizing. Although some members of the HRO group are exploring meaning, action, and agency in several interesting dimensions, their work addresses the issue of safety only piecewise (Weick and Roberts 1993). For the present purpose, it is more appropriate to continue to be descriptive, to focus attention on organizations that are broadly and generally considered to be safe (in some cases remarkably and even surprisingly so), and to describe what are thought to be the most relevant, unique and important safetyrelated observations that have come out of the group's work rather than trying to generate a more comprehensive study.

\subsection{Learning}

In order to maintain safety in a dynamic and changing organizational setting and environment, system operators and managers must continue to learn. By what standards can that learning be gauged? There is considerable individual learning, and some of the organizations consider it to be central to their performance (Rochlin et al. 1987). What is less clear without a theory to determine the appropriate level of agency is whether that translates into organizational or institutional learning. In his analysis of risk and social learning, Wynne (1992) has invoked a parallel with the reflexive and self-conscious model of organizational learning developed by Schön and Argyris (1978), adding further that institutions that learn do not exclude their own structure and social relations from the discourse. Instead, Wynne asserts, they eschew narrow definitions of efficiency or risk and embrace multiple rationalities:

'It is the connected dimensions, of self-exploration combined with developing relations with others and their forms of knowledge and experience, which constitute the crucial reflexive and interactive learning. This has no preordained or guaranteed direction; indeed, it needs recognition of the indeterminacy of values, identities, and knowledges in order to be possible (Wynne 1992: 293). (emphasis in the original)'

The essential indeterminacy of values, identities and knowledge is precisely what characterizes the safety learning seen in these organizations, with the added dimension that it is not just the organization as a whole, but differently positioned actor-groups who are learning, and that each learns different things at different times from different signals sited or anchored in different operational contexts. Nor are the signals themselves always obvious to even a trained observer. Many of these organizations are in a situation where they cannot indulge in trial and error. Because their first error might be their last trial, they learn not only from near misses, but even from situations which they evaluate as 
having had the potential to evolve into near misses. That learning is inherently reflexive, since the ability to identify such situations is in itself part of the constructed ambience of safe operation.

\subsection{Duality}

A closely related observation about the organizations studied by the HRO group was the ability simultaneously to maintain multiple representations of the state and structure of operations. The most extreme circumstance was that of naval flight operations, where different organizational subunits collected in different overlapping and interpenetrating ad-hoc groups to sketch out and rehearse latent scripts for response to future possibilities that would require rapid and practiced co-ordination (Rochlin et al. 1987). However, even the simplest of these organizations showed a remarkable ability simultaneously to act in the dual frame of contrasting representations - in some cases that of safety in the midst of risk, in others that of independence of action in a context of exceptionally close and detailed regulation (Rochlin and von Meier 1994, Bourrier 1996). The HRO group's first conclusion was that this was a means of learning through dialectic. However, when the duality was pointed out to those who were interviewed, they saw the frames as reinforcing rather than standing in opposition. They do not themselves note the organizational contradictions; and although one can elicit the dialectical tension by varying the framing and wording of specific questions put to them, the interviewees resist that interpretation when it is put to them.

The operators in the HRO system resemble commercial pilots in maintaining that reliably safe operation depends on treating their operational environment not only as inherently 'risky', in the sense of embodying the possibility for error, but also as an actively hostile one in which error will seek out the complacent (Rochlin 1993). Confidence in the equipment and the training of the crew does not diminish the need to remain alert for signs that a circumstance exists or is developing in which that confidence is erroneous or misplaced (Gras et al. 1994, Rochlin 1993). This also extends to interactions with regulators and others who impose interactive oversight. US nuclear plant operators often describe regulation as intrusive, distracting and unnecessarily detailed, but they also argue that it is necessary and important (La Porte and Thomas 1995). Clearly, even external constraints such as regulation become incorporated as essential rather than contingent, as elements from which collective behaviour is constructed and not one that is superimposed, as are most attempts at instilling safety culture. The assumption of responsibility in these organizations is more than an expression of individual or collective action, it is a constitutive property that enters both as agency and as structure.

\subsection{Communication}

In work by the HRO group on aircraft carrier flight operations, a constant stream of traffic was found to take place simultaneously over multiple communication links during flight operations, even when the level of operational activity was low (Rochlin et al. 1987). By comparing it with other similar, if less dramatic, activities taking place elsewhere on the ship during critical operations, it was found that the constant chatter was an important means for maintaining the integration of the collectivity and reassuring all participants of its status. Similar behaviour was also seen in nuclear power plant control rooms (Rochlin and von Meier 1994). Air traffic control is radically different, in that controllers interact more with pilots than with each 
other. Nevertheless, Sanne's (1996) analysis of extensive video and audio recording of conversations between controllers and pilots at a Scandinavian control centre also displayed many interactions that were not part of the formal control process, but meant to nurture communication and co-operation between controllers and pilots. Their structure and content were shaped to a great extent by the recognition of all parties concerned that safety was indeed a collective property that 'emerged' from the interaction between them.

Similar findings have long been reported in the literature. The importance of control room inter-communication is a fundamental finding of the Westinghouse group and others (Roth et al. 1992). The 'social organizational safety' described by Janssens et al. (1989: 124) depends on the 'spontaneous and continuous exchange of information relevant to normal functioning of the system in the team, in the social organization'. Such inter-communication was therefore identified as a primary goal of safety training programmes for operators. However, it has an additional safety function as well. In the HRO studies, every operational team that was interviewed pointed out the importance of maintaining a free flow of information at all times, not only to keep the team integrated but also because experienced operators monitoring the flow of what often seems to be trivial or irrelevant information can often discern in it warning signs of impending trouble.

\subsection{The locus of responsibility}

Schulman (1996) has suggested a frame for understanding the importance of collective behaviour in defining operational safety by noting a sharp contrast between organizations in which hero stories were prevalent and welcome and those in which such stories are rare and not encouraged. Organizations such as militaries and fire departments emphasize extraordinary individual performance and rapid response; hero stories become epics that define organizational culture as well as being an effective means for organizational learning and the maintenance of cumulative knowledge. In contract, 'hero' is a critique in air traffic control and nuclear power, describing an operator who will act with little regard for the collectivity. Safety is sought through collective (inter)action, through shared knowledge and responsibility. Organizational learning is formalized, and cumulative knowledge transmitted not through legends or narratives but through the creation and modification of formal procedures.

The two differing representations of operator responsibility and operational safety lead to contrasting sets of social and individual behaviour. The first establishes a context in which responsibility for performance of those tasks that are not routine (particularly those that entail the most risk, whether to the public or to the organization) rests primarily with individuals, potential heroes to be sought out and nurtured; what lends confidence in collective performance is the belief that any contingency will be met by one or the other. The second rejects such an approach as dangerous, especially for those tasks that present the greatest potential for harm. Threats are to be identified, scoped, and routinized; when unexpected events do occur, they must be responded to through collective action; heroic individual initiative is a threat, not an opportunity. In both cases, safe operation is a positive outcome, and the constructed narrative is one of organizational rather than individual performance.

This analysis builds on and reinforces two earlier conclusions of the HRO research. First, that reliable organizations tend to reward the reporting of error and 
the assumption of responsibility for error; second, that even when human error does occur, the root cause is considered to lie with the organization as a whole rather than being displaced onto the erring task group or individual (Weick 1987, Weick and Roberts 1993). The latter behaviour is quite remarkable, in that it spans analytic conclusions ranging from taking overall responsibility for poor training or bad working conditions to a lack of collective wisdom in putting an unqualified individual into a responsible position. Even the extreme action of explicitly blaming the individual for malfeasance does not, in this view, absolve the organization of responsibility for the outcome. Agency has become detached from individual actors and work groups and devolved upon the collectivity, in ways that are not yet fully understood.

\section{Safety as myth and ritual}

In search of analogies to explain these observations, the group follows Reason (1995) and examines safety by comparing it with more common definitions of health. If one takes perceived risk as a constructed measure of the potential for error, safety stands in the same relationship to error, and risk, as health does to illness, and the potential for being unwell. Reason further points out that health can be though of as 'an emergent property inferred from a selection of physiological signs and lifestyle indicators' (Reason 1995: 1716). He then constructs an analogy with complex, hazardous systems, pointing out that assessing an organization's 'safety health' resembles the medical case in that it requires sampling from a potentially large number of indicators. Although Reason's argument then proceeds in a more positivistic direction towards the development of separable indicators than the social constructivist perspective followed here, the analogy is nevertheless apt.

This sharpens the point about taking safety to be a positive construct and not just as the opposite of risk. Defining an organization as safe because it has a low rate of error or accident has the same limitations as defining health in terms of not being sick. When a person characterizes their medical state as one of being 'healthy', they usually mean far more than not being ill, as is increasingly recognized by those who have adopted the term 'wellness' rather than 'health' (Edlin et al. 1996). Other factors that may enter include a calculation that they are not at the moment at any risk of being ill, or have a cushion or margin of protection against illness, that is having a low potential for being unwell. In most cases, wellness goes beyond health in expressing a state of being with many dimensions that is not entirely about the clinical state of the physical body, but a story about the relationship of the individual's mind and body with both real and perceived social and physical environments. Indeed, there is a body of literature that argues that perceptions such as being able to control one's environment promote being well, even when measured as 'good health' by objective indicators, an observation that has recently been emphasized by noting that even placebos can have positive effects (Harrington 1997).

Wellness is in some sense a story one tells about one's self and one's relation to others and the world. Safety is in some sense a story a group or organization tells about itself and its relation to its task environment. The origins of both do not lie solely with indicators or performance. Meyer and Rowan (1977) have noted that there is a correlation between elaborate displays of confidence, satisfaction, and good faith and the institutionalization of myth and ritual. Moreover, these are in most cases more than affirmations of institutionalized myths; they are the basis for a commitment to support that representation by collective action ('t Hart 1993 ). 
Putting these together with the HRO group's observations, it is argued that a collective commitment to safety is an institutionalized social construct. The organizations observed not only perform well, but weave into their operations the kinds of rituals and stories that serve to orally transmit operational behaviour, group culture and collective responsibility. Since these arise from the organization's perpetual reaffirmation of its commitment to safety not only as an institutionalized collective property but also as a constant process of re-enactment of its own stories, it is in many ways a dynamic, interactive, and therefore inter-subjective process that can only be nurtured and maintained if the means and modes of social interaction are preserved and reinforced.

\section{The search for safety is not just a hunt for error}

The central role of free information flows, learning, and responsibility as elements for constructing a cognitive frame for safe operations has long been a source of concern in critical studies of control room procedures and airline cockpits. The fundamental question has been whether, or to what extent, automation and other technical strategies aimed at reducing the rate of operational error tend to be reductionist and overly objective, focusing on specifics of individual or small group behaviour, and therefore often pay insufficient attention to representations, social constructs, and other more subjective collective behaviour (Bainbridge 1987, W eick 1987 ). There are already examples in the literature of cases where positivist efforts to 'improve' the operating environment of control rooms have been antithetical to norms and routines that support and integrate the team (Roth et al. 1992, Gras 1993 ). These are not always direct, but may also arise from automation put into place for other reasons, such as plant or process efficiency.

The case of cockpit automation has long been raised as a prime example of the potential for reducing rather than increasing safety by interfering with the cognitive processes of integration (Anon. 1992, Gras et al. 1994). Others have shown that as the level of plant or process automation increases, the role of the operator is more and more that of a supervisory controller of the equipment rather than of the plant, which can interfere with operators' sensitivity to warning signs, or ability to respond to unexpected events (Zuboff 1984, Rochlin 1997). Similar, and more general arguments have been made from the perspective of cognitive ergonomics (Hollnagel 1997). Modifying equipment, task environment or organization in the name of reducing 'risk' without fully understanding social and cultural factors may well result in increasing operational error by interfering with the less explicitly observable processes by which safety is created and maintained (Dougherty 1993).

Perrow and other primarily structural analysts who apply the normal accident framework to the study of complex tightly-coupled technical systems have frequently warned that safety devices or other levels of additional technical redundancy can increase overall complexity and coupling, resulting in systems that are more prone to error than they were previously (Perrow 1984, Sagan 1993). In the HRO work, operators of comparatively reliable systems were self-consciously aware of this problem; several instances were found where operators opposed further introduction of supposedly error-reducing procedures or technologies for precisely those reasons. The argument presented here takes that a step further, building on the argument of reliability theorists that some organizations possess interactive social characteristics that enable them to manage such complex systems remarkably well, and the further observation that we do not know enough about either the construction or the 
maintenance of such behaviour to be confident about its resilience in the face of externally imposed changes to task design or environment.

The concern is that efforts aimed at improving performance or reducing human error, when insensitively or clumsily done, can decouple the socially constructed image of safe operation from the reality, transforming an organization that can reliably judge whether it is in a 'safe' state of operation to one that cannot. This is a familiar problem to those who have followed the ongoing controversies over the safety and reliability of complex technical operations. Operators, teams, and organizations may overestimate their knowledge, misread the state of their system(s), or simply try to do more than they are capable of because it is expected of them (Rochlin 1997). 'Hero-seeking' organizations are known to be particularly susceptible to such representational errors, but similar cases have been observed in hero-avoiding systems where operators became so confident in their equipment (or organization ) that they ignored procedures or become inattentive even to manifestly displayed signals. There are cases where operating teams assumed that a clear indication of malfunction was read as a problem with the instrument and not the plant, and other cases where the possibility of error at all was simply never considered (Sagan 1993, Vaughan 1996).

Throughout this research, it was observed again and again, in different contexts and in different manifestations, that maintenance of a high degree of operational safety depends on more than a set of observable rules or procedures, externally imposed training or management skills, or easily recognized behavioural scripts. While much of what the operators do can be formally described one step at a time, a great deal of how they operate, and, more importantly, how they operate safely, is 'holistic', in the sense that it is a property of the interactions, rituals, and myths of the social structure and beliefs of the entire organization, or at least of a large segment of it. Moreover, at least in the case of nuclear plants, air traffic control, and aircraft carrier flight operations, it is not just an intersubjective social construct within the group but 'generically' subjective in that it reflexively incorporates group performance as part of the structure of the relationship between task, task environment, task group and organization. The more technically complex the system (and nuclear plants probably do occupy that end of the spectrum) the less possible it is to decompose the interlocking set of organizationally and technically framed social constructs and representations of the plant in search of the locus of operational safety (La Porte and Consolini 1991).

Analysing the more subjective and social dimensions is difficult because so much of our theory, and our research experience, remains grounded in structureoriented, rational-action, and task-centred approaches that are fundamentally oriented towards measurable indicators of the interfaces and relationships between observable organizational characteristics, the performance of individual workers and the nature of their machinery. This extends not only to technical model building and organizational analysis, but also to human-technical interactions, psychological theories of framing and scenario-building, and even cognitive analysis. Ritualization, seemingly non-functional displays, and expressions of confidence that are not grounded in empirically-determinable observables are all too often disregarded, or, worse, trampled on or overwritten in an effort to impose theoretically-argued strategies that are argued to be 'functional' solely in terms of abstractly constructed models and formalized indicators and observables. 
The challenge is to gain a better understanding of the interactive dynamics of action and agency in these and similar organizations and the means by which they are created and maintained. These arise as much from interpersonal and intergroup interaction as from more commonly studied interactions with external designers and regulators. The interaction is a social construction anchored in cultural dynamics, therefore there are wide variances in its manifestation even for similar plants in roughly similar settings. An even greater challenge will be to identify rules that correlate constructions of operational safety with empirical observations of performance, so as to separate those organizations that construct a representational framework of safety that is accurate from those who only construct the representation, and not the safety.

\section{Conclusion}

The purpose of this paper has not been to recapitulate the findings of the HRO group, nor to continue the argument about the relationship between complexity, coupling and reliability. The organizations that have been observed do perform both reliably and effectively at a level that cannot be explained by most of the literature on organizational reliability and performance in the face of high complexity, high coupling and very high potential consequences of error. What the author argues here is that a broader range of constructivist social approaches is needed if we are to advance our understanding of how these organizations maintain safety in the face of such challenges, and how institutional behaviour that encompasses such nonquantifiable and collective properties as enacted drama and ritualization of myth contributes to safety - as a positive construct and not just the control of risk.

To those of us trained in formal and rational approaches to analysing organizational performance, such an approach may seem strange, even unprofessional, a prescription so far removed from the usual canons of analysis that some will argue that it can have no generalizable meaning or prescriptive power (Balfour and Mesaros 1994). Yet, throughout this research, it was observed again and again, in different contexts and in different manifestations, that 'operational safety' is not capturable as a set of rules or procedures, of simple, empirically observable properties, of externally imposed training or management skills, or of decomposable cognitive or behavioural frames. While much of what the operators do can be empirically described and framed in positivistic terms, a great deal of how they operate, and, more importantly, how they operate safely, is a property of the interactions, rituals, and myths of the social structure and beliefs of the entire organization, or at least of a large segment of it. Moreover, at least in the case of nuclear plants, air traffic control, and aircraft carrier flight operations, safety is an emergent property not just at the individual but also at the inter-group level.

However intersubjective, socially constructed and collective these descriptors may be, they are central to understanding the role of agency in providing safety as a positive effect that is centrally important in understanding the reliable operation of complex, potentially hazardous socio-technical systems. To the extent that regulators, systems designers, and analysts continue to focus their attention on the avoidance of error and the control of risk, and to seek objective and positivistic indicators of performance, safety becomes marginalized as a residual property. This not only neglects the importance of expressed and perceived safety as a constitutive property of safe operation, but may actually interfere with the means and processes by which it is created and maintained. It is 
therefore important in practice, as well as in theory, to encourage continued and expanding research and enquiry into safety as an expression of myth and ritual, of agency as well as structure, and not just as a measurable, statistical property or organization, a reporting system for work team errors, or a way of expressing the avoidance of consequential accidents.

\section{References}

't Hart, P. 1993, Symbols, rituals and power: the lost dimensions of crisis management, Journal of Contingencies and Crisis Management, 1, 36-50.

AкRICH, M. 1992, Beyond social construction of technology: the shaping of people and things in the innovation process, in M. Dierkes and U. Hoffmann (eds), New Technology at the Outset: Social Forces in the Shaping of Technological Innovations (Frankfurt and New York: Campus/Westview ), $173-190$.

Anon. 1992, Automated cockpits: keeping pilots in the loop, Aviation Week \& Space Technology, 23 March, $48-71$.

Bainbridge, L. 1987, Ironies of automation, in J. Rasmussen, K. Duncan, and J. Leplat (eds), Technology and Human Error (New York: John Wiley), 271-286.

Balfour, D. L. and Mesaros, W. 1994, Connecting the local narratives: public administration as a hermeneutic science, Public Administration Review, 54, 559-564.

Bourrier, M. 1996, Organizing maintenance work at two American nuclear power plants, Journal of Contingencies and Crisis Management, 4, 104-112.

Burns, T. R. and Dietz, T. 1992, Technology, sociotechnical systems, technological development: an evolutionary perspective, in M. Dierkes and U. Hoffmann (eds), New Technology at the Outset: Social Forces in the Shaping of Technological Innovations (Frankfurt and New York: Campus/Westview), 207-238.

Christensen, S., Karnoe, P., Pedersen, J. S. and Dobbin, F. 1997, Action in institutions, American Behavioral Scientist, 40, Special Issue.

Dougherty, E. M. 1993, Context and human reliability analysis, Reliability Engineering and System Safety, 41, $25-47$.

Edlin, G., Golanty, E. and Brown, K. M. (eds) 1996, Health and Wellness (Sudbury, MA; Jones and Bartlett).

Emirbayer, M. and Goodwin, J. 1994, Network analysis, culture, and the problem of agency, American Journal of Sociology, 99, $1411-1454$.

Gras, A. 1993, Grandeur et Dépendance: Sociologie Des Macro-systèmes Techniques (Paris: Presses Universitaires de France).

Gras, A., Моricot, C., Рoirot-Delpech, S. L. and Scardigli, V. 1994, Faced with Automation: the Pilot, the Controller, and the Engineer, translated by J. Lundsten (Paris: Publications de la Sorbonne).

Harrington, A. (ed.) 1997, The Placebo Effect: An Interdisciplinary Orientation (Cambridge, MA: Harvard University Press).

Hollnagel, E. 1997, Cognitive ergonomics: it's all in the mind, Ergonomics, 40, 1170-1182. Hutchins, E. 1995, Cognition in the Wild (Cambridge, MA: MIT Press).

International Nuclear Safety Advisory Group [INSAG] 1991, Safety Culture, Safety series No. 75-INSAG-4 (Vienna: International Atomic Energy Agency).

Janssens, L., Grotenhus, H., Michels, H. and Verhaegen, P. 1989, Social organizational determinants of safety in nuclear power plants: operator training in the management of unforeseen events, Journal of Organizational Accidents, 11, $121-129$.

Krimsky, S. and Golding, D. (eds) 1992, Social Theories of Risk (Westport, CT: Praeger).

La Porte, T. R. 1996, High reliability organizations: unlikely, demanding, and at risk, Journal of Contingencies and Crisis Management, 4, 60-71.

La Porte, T. R. and Consolini, P. M. 1991, Working in practice but not in theory: theoretical challenges of 'high-reliability organizations', Journal of Public Administration Research and Theory, 1, $19-47$.

La Porte, T. R. and Thomas, C. W. 1995, Regulatory compliance and the ethos of quality enhancement: surprises in nuclear power plant operations, Journal of Public Administration Research and Theory, 5, 109-137. 
Meyer, J. W. and Rowan, B. 1977, Institutionalized organizations: formal structure as myth and ceremony, American Journal of Sociology, 83, 340-363.

Perrow, C. 1984, Normal Accidents: Living with High-risk Technologies (New York: Basic Books).

Pidgeon, N. F. 1991, Safety culture and risk management in organizations, Journal of CrossCultural Psychology, 22, $129-140$.

Reason, J. 1995, A systems approach to organizational error, Ergonomics, 39, 1708 - 1721.

Rochlin, G. I. 1993, Defining high-reliability organizations in practice: a taxonomic prolegomenon, in K. H. Roberts (ed.), New Challenges to Understanding Organizations (New York: Macmillan), $11-32$.

Rochlin, G. I. 1997, Trapped in the Net: The Unanticipated Consequences of Computerization, (Princeton: Princeton University Press).

Rochlin, G. I. and von MeIER, A. 1994, Nuclear power operations: a cross-cultural perspective, Annual Review of Energy and the Environment, 19, 153-187.

Rochlin, G. I., La Porte, T. R. and Roberts, K. H. 1987, The self-designing high-reliability organization: aircraft carrier flight operations at sea, Naval War College Review, 40, $76-90$.

Roth, E. M., Mumaw, R. J. and Stubler, W. F. 1992, Human factors evaluation issues for advanced control rooms: a research agenda, in E. W. Hagen (ed.), IEEE Fifth Conference on Human Factors and Power Plants (New York: Institute of Electrical and Electronics Engineers), 254-260.

SAGAn, S. D. 1993, The Limits of Safety: Organizations, Accidents, and Nuclear Weapons (Princeton: Princeton University Press).

SAnNe, J. M. 1996, Air traffic control as situated action: a preliminary study, Proceedings of the Conference on Social Studies of Science and Technology, Charlottesville, VA, October 1995.

Schön, D. A. and Argyris, C. 1978, Organizational Learning: A Theory of Action Perspective (New York: Addison-Wesley).

Schulman, P. R. 1996, Heroes, organizations, and high reliability, Journal of Contingencies and Crisis Management, 4, $72-82$.

Sewell, W. H., Jr. 1992, A theory of structure: duality, agency, and transformation, American Journal of Sociology, 98, $1-29$.

Simpson, R. 1996, Neither clear nor present: the social construction of safety and danger, Sociological Forum, 11, 549-562.

Slovic, P. 1992, Perception of risk: reflections on the psychometric paradigm, in S. Krimsky and D. Golding (eds), Social Theories of Risk (Westport, CT: Praeger), 117-152.

Vaughan, D. 1996, The Challenger Launch Decision: Risky Technology, Culture, and Deviance at NASA (Chicago: University of Chicago Press).

Weick, K. E. 1987, Organizational culture as a source of high reliability, California Management Review, 29, $112-127$.

Weick, K. E. and Roberts, K. H. 1993, Collective mind in organizations: heedful interrelating on flight decks, Administrative Science Quarterly, 38, 357-381.

Wiley, N. 1988, The micro-macro problem in sociological theory, Sociological Theory, 6, $254-261$.

Wynne, B. 1992, Risk and social learning: reification to engagement, in S. Krimsky and D. Golding (eds), Social Theories of Risk, (Westport, CT: Praeger), 275-300.

Zuboff, S. 1984, In the Age of the Smart Machine: the Future of Work and Power (New York Basic Books). 
Copyright $\odot 2003$ EBSCO Publishing 